\title{
Neutrophils use selective autophagy receptor p62/SQSTM1 to target Staphylococcus aureus for degradation in vivo in zebrafish
}

\author{
Josie F Gibson ${ }^{1,2,3,4,5}$, Tomasz K Prajsnar ${ }^{1,2,6}$, Christopher J Hill ${ }^{5}$, Amy K Tooke ${ }^{5}$, Justyna J \\ Serba $^{1,2}$, Rebecca D Tonge ${ }^{7}$, Simon J Foster ${ }^{4,5}$, Andrew J Grierson ${ }^{2,7}$, Philip W Ingham ${ }^{3,8 \#}$,

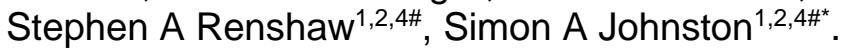

\footnotetext{
1. Department of Infection, Immunity and Cardiovascular disease, Medical School, University of Sheffield, S10 2RX UK.

2. The Bateson Centre, University of Sheffield, Sheffield, S10 2TN, UK

3. Institute of Molecular and Cell Biology, Agency of Science, Technology and Research (A-Star), Singapore 138673

4. Florey Institute, University of Sheffield, Sheffield, United Kingdom, S10 2TN

5. Department of Molecular Biology and Biotechnology, University of Sheffield, S10 2TN, UK

6. Institute Biology Leiden, Leiden University, Leiden, The Netherlands

7. Sheffield institute for Translational Neuroscience, Department of Neuroscience, University of Sheffield, S10 2HQ, UK

8. Lee Kong Chian School of Medicine, Nanyang Technological University, Singapore 636921
}

${ }^{*}$ Correspondence: s.a.johnston@sheffield.ac.uk \#Joint senior author

\section{Abstract}

Autophagy leads to degradation of cellular components and has an important role in restricting intracellular pathogens. Autophagy receptors, including p62, target invading intracellular pathogens to the autophagy pathway for degradation. Staphylococcus aureus is a significant pathogen of humans and often life-threatening in the immunocompromised. Increasing evidence demonstrates that $S$. aureus is an intracellular pathogen of immune cells and may use neutrophils as proliferative niche but the intracellular fate of $S$. aureus following phagocytosis by neutrophils has not previously been analysed in vivo. In vitro, p62 is able to co-localise with intracellular Staphylococcus aureus, but whether p62 is beneficial or detrimental in host defence against $S$. aureus in vivo had not been determined.

Here we use zebrafish to determine the fate and location of $S$. aureus within neutrophils throughout infection. We show that Lc3 and p62 recruitment to phagocytosed $S$. aureus is altered depending on the bacterial location within the neutrophil. We also show rapid Lc3 marking of bacterial phagosomes within neutrophils which may be associated with subsequent bacterial degradation. Finally, we find that p62 is important for controlling cytosolic bacteria demonstrating for the first time a key role of p62 in autophagic control of $S$. aureus in neutrophils. 


\section{Introduction}

Autophagy (macroautophagy) is a process of cellular self-degradation, in which damaged or redundant cellular components are taken into an autophagosome and subsequently trafficked to the lysosome for degradation; these degraded components can then be recycled for alternative uses by the cell (Mizushima et al., 2008; Tanida, 2011). During infection, autophagy is used by host cells to degrade invading pathogens, a process termed xenophagy (Gatica, Lahiri and Klionsky, 2018; Sharma et al., 2018).

Autophagy is considered largely non-selective of the cargo to be degraded, classically being induced by starvation conditions. However, selective autophagy is a process that enables specific cargo to be directed into the autophagy pathway, which can be used to target invading pathogens. Selective autophagy uses autophagy receptors (ARs), proteins that interact with both autophagy machinery and the cargo to be degraded (Popovic and Dikic, 2012; Rogov et al., 2014). Many ARs are involved in targeting invading pathogens, including p62 (also named sequestosome 1 (SQSTM1)), neighbour of Brca1 gene (NBR1), optineurin (OPTN) and nuclear dot protein 52 (NDP52) (Farré and Subramani, 2016).

Loss of autophagy function, for example through mutations in key autophagy genes, can increase the risk of infection with intracellular pathogens (Levine, Mizushima and Virgin, 2011). It is well established that pathogen presence can induce host cell autophagy and that pathogens can be degraded by this pathway. Intracellular pathogens such as Mycobacterium marinum, Shigella flexneri and Listeria monocytogenes (Mostowy et al., 2011; Zhang et al., 2019) can be targeted by ARs for degradation. Conversely, pathogens have evolved to be able to block or subvert immune defences, and autophagy is no exception. Indeed, many bacterial pathogens are able to inhibit induction of autophagy or to reside within the autophagy pathway by preventing lysosomal fusion, or even avoid making any contact with autophagic machinery (Deretic and Levine, 2009). In some cases it is beneficial to the pathogen to upregulate the autophagy pathway, for example Legionella pneumophila, Coxiella burnetii and Salmonella enterica serovar typhimurium (Hernandez et al., 2003; Amer and Swanson, 2005; Gutierrez et al., 2005). The outcome of host-cell autophagy therefore differs between various invading pathogens.

Staphylococcus aureus is a bacterial pathogen that is able to reside within neutrophils as an intracellular niche (Thwaites and Gant, 2011; Prajsnar et al., 2012). Autophagy has been implicated in $S$. aureus infection but there are conflicting reports suggesting autophagy might be either beneficial (Schnaith et al., 2007) or detrimental for S. aureus (Neumann et al., 2016). 
Intracellular pathogens, including $S$. aureus, are able to escape the phagosome into the cytosol (Bayles et al., 1998), likely through toxins secreted by the bacteria or membrane rupture due to bacterial growth. Once in the cytosol, bacteria can be ubiquitinated and targeted by ARs (Farré and Subramani, 2016). Indeed, p62 in fibroblasts and epithelial cells has been shown to localise to cytosolic $S$. aureus leading to autophagsome formation in vitro (Neumann et al., 2016; Singh et al., 2017). Therefore, we investigated whether p62 recruitment is employed by neutrophils in $S$. aureus infection and what influence selective autophagy has on infection outcome in vivo.

In order to examine the role of neutrophil autophagy in $S$. aureus infection, we compared the fate of bacterial cells following Lc3 and p62 recruitment. We tested the role of p62 in pathogen handling in vivo, using the genetic tractability of the zebrafish to create a neutrophil-specific p62-GFP transgenic reporter, and a $p 62$ activity deficient mutant. With this approach we show that p62 is recruited to cytosolic $S$. aureus and disruption of p62 expression or function adversely affects $S$. aureus infection outcome.

\section{Results}

\section{Staphylococcus aureus location within neutrophils changes throughout infection}

Autophagy responses have been demonstrated to change throughout the progression of infection. Targeting of pathogens by autophagy receptors is likely to occur at later time points in infection. Therefore, to determine the fate and location of $S$. aureus in neutrophils during infection, $S$. aureus expressing mCherry was inoculated and imaged at early ( 2 to 5 hours post infection (hpi)) and late (24 to 28hpi) time points. Initially, the well-established $\operatorname{Tg}$ (mpx:eGFP)i114 line that specifically marks neutrophils with EGFP (Renshaw et al., 2006) was used to analyse the fate of intracellular $S$. aureus throughout infection. Imaging throughout the whole organisms demonstrated a marked reduction in the number of bacterial cells within individual neutrophils), and that the number of neutrophils containing $S$. aureus, between 2 and 24 hours post infection (Figure 1A, B). This suggested to us that neutrophils were able to degrade intracellular $S$. aureus effectively throughout infection. Indeed, video timelapse of $T g$ (mpx:eGFP)i114 larvae infected with mCherry S. aureus demonstrated that bacteria can be effectively degraded by the host neutrophils (Figure 1C), although in other cases the bacterial infection is not controlled (Supplementary Figure 1A).

We next sought to determine the location of bacteria, and relationship to autophagic machinery, within neutrophils. To do this we used the newly generated Tg(lyz:RFP-GFPLc3)sh383 (Prajsnar et al., 2019). We first confirmed that, in the caudal hematopoietic tissue 
(CHT), the infection dynamics were similar to the $T g(m p x: e G F P) i 114$ line, with a significant reduction in intracellular bacteria by $26 \mathrm{hpi}$, indicating bacteria are efficiently controlled and a significant reduction in infected neutrophils was observed (Figure 1D). Importantly, the number of neutrophils analysed in the $\mathrm{CHT}$, used for analysis throughout this study, did not significantly change between 2dpf and 3dpf (Supplementary Figure 1B), demonstrating that the change in proportions of infected neutrophils is not due to a large increase in neutrophil number between these time points. The labelling of $S$. aureus containing vesicles enabled the identification of intracellular bacteria that were within a vesicle (Figure 1E) or free in the cytosol (Figure 1F), as well as non-labelled vesicles, or vesicles marked with Lc3 puncta (Supplementary Figure 1C, D). We found that the proportion of bacteria within vesicles was significantly reduced over time post-injection, whereas the number of bacteria within the cytosol remains relatively constant at a low level, despite becoming proportionally higher relative to vesicular bacteria (Figure 1G). Thus, S. aureus phagocytosed by a neutrophil are initially located in a phagocytic vesicle and are subsequently degraded. However, a smaller proportion of $S$. aureus is able to survive to later infection time points, and these predominantly reside in the cytosol.

\section{Generation and characterisation of an in vivo neutrophil GFP-p62 reporter line}

A previous study identified colocalisation of p62 with S. aureus in non-immune cells (Neumann et al., 2016). Our findings demonstrated a small but significant population of bacteria that were cytosolic, and therefore a possible target for p62 binding. Accordingly, we generated a transgenic neutrophil-specific p62 reporter zebrafish line to examine whether p62 and intracellular pathogens are co-localised in vivo. We used GFP fused via a small linker region to the $\mathrm{N}$-terminus of $p 62$ in order to produce a fluorescently marked fusion protein expressed within neutrophils via the lysozyme C (lyz) promoter (Yang et al., 2012). Using larvae with double labelled neutrophils, we were able to identify GFP expressing cells from the Tg(lyz:eGFP-p62)i330 reporter line (hereafter called GFP-p62 reporter) also expressing mCherry (Tg(lyz:nfsB-mCherry)sh260) (Buchan et al., 2019) in 98\% of neutrophils observed (Supplementary Figure 2A-C).

We next examined whether the GFP-p62 protein is able to function as expected. Interestingly, in the double labelled larvae, GFP puncta but not mCherry puncta were seen (Supplementary Figure 2D). Similar p62 puncta that required UBD to function have been observed in vitro for endogenous p62 (Bjørkøy et al., 2005). To test whether the GFP-p62 puncta observed in the GFP-p62 reporter line respond as expected, GFP-p62 reporter larvae were treated with autophagy inhibitor Bay-K8644 (known to block autophagy in zebrafish; (Williams et al., 2008). As expected, there was a significant increase in the number of neutrophils which contained GFP-p62 puncta following Bay-K8644 treatment in comparison to non-treated controls 
within individual neutrophils as expected for endogenous p62 (Supplementary Figure 2F). This suggests that the GFP-p62 puncta are not being processed through autophagy accumulate within the cell, as reported for endogenous p62 (Bjørkøy et al., 2009).

As we had done for neutrophils and Lc3 positive vesicles, we examined the location of $S$. aureus throughout infection with our GFP-p62 reporter for consistency with Tg(mpx:eGFP)i114 and Tg(lyz:RFP-GFP-Lc3)sh383 (Figure 1). We found that there was a comparable reduction in the number of bacteria observed within neutrophils at 26hpi in comparison to $2 \mathrm{hpi}$ (Supplementary Figure $3 \mathrm{~A}$ ) and a reduction in the number of infected neutrophils from $2 \mathrm{hpi}$ to $26 \mathrm{hpi}$ (Supplementary Figure $3 \mathrm{~B}$ ). This suggested that neutrophils were efficiently degrading these bacteria, in agreement with Figure $1 \mathrm{C}$.

Cytosolic bacteria are a possible target for p62 and $S$. aureus has previously been visualised within the cytosol of a neutrophil from murine infection studies (Gresham et al., 2000).To identify $S$. aureus in the cytosol in our in vivo experiments in zebrafish we looked for regions of the cytosol that co-localised with S. aureus but without a reduction of GFP signal indicating a vacuole excluding the surrounding cytosol (containing GFP). We first confirmed we were able to clearly observe phagosomes containing bacteria with low GFP fluorescence consistent with $S$. aureus containing vacuoles, where host cell cytoplasm containing GFP, was excluded (p62GFPlow, Supplementary Figure 3C). To further evidence this analysis, we determined that vesicles containing $S$. aureus visualised by TEM were empty of cellular components, in comparison to the cytosol (Supplementary Figure 3D), suggesting GFPlow areas represent vesicles. Finally, we looked for function differences consistent with the presence of a phagosomal membrane in GFPlow regions by examining $\mathrm{pH}$ differences using the $\mathrm{pH}$ sensitive dye pHrodo. We found examples of low $\mathrm{pH}$ in vesicles correlating with low cytoplasmic fluorescence (Supplementary Figure 3E), again suggesting GFPlow areas represent vesicles. Having characterised features consistent with a $S$. aureus containing vacuoles we were able to assign a subset of bacteria as being in either a damaged phagosome or located in the cytosol (p62GFPhigh, Supplementary Figure 3F); for the purpose of this study we are defining these bacteria as cytosolic as they are accessible to cytosolic proteins. We then assigned the cellular location of $S$. aureus by these features at $2 \mathrm{hpi}$ and $26 \mathrm{hpi}$. We determined that the proportion of $S$. aureus within vesicles was significantly reduced by $26 \mathrm{hpi}$ (Supplementary Figure $3 G$ ), and that the number of bacteria within the cytosol is similar at both time points, in agreement with our Tg(lyz:RFP-GFP-Lc3)sh383 data (Figure 1).

\section{Lc3 and p62 are recruited to Staphylococcus aureus within neutrophils}

We determined that GFP-p62 puncta co-localise with $S$. aureus either marking a vesicle containing $S$. aureus (Figure $2 \mathrm{~A}$, supplementary video 1 ), or directly in contact with $S$. aureus 
located in the cytosol (Figure 2B, supplementary video 2). For puncta marking $S$. aureus in vesicles, no difference in the proportion of vesicles marked was observed at 2 or $26 \mathrm{hpi}$, although the actual number of puncta marking was dramatically reduced by $26 \mathrm{hpi}$ (Figure $2 \mathrm{C}$ ) as most bacteria had already been degraded. GFP-puncta marking bacteria in the cytosol were decreased at 26hpi (Figure 2D), as expected given that p62 is degraded with the cargo targeted for degradation (Bjørkøy et al., 2009). We could show cytosolic GFP-p62 puncta were modulated by autophagy machinery-targeting drugs (Supplementary Figure 2E-F). In further agreement with this, comparison between infected and uninfected neutrophils showed there was no difference in the number of cytoplasmic GFP-p62 puncta at 2hpi but a significant reduction by $26 \mathrm{hpi}$ (Figure $2 \mathrm{E}, \mathrm{F}$ ), indicating these puncta are modulated by $S$. aureus infection. We next examined whether Lc3 is able to localise to vesicular and cytosolic $S$. aureus. At $2 \mathrm{hpi}$ and $26 \mathrm{hpi}$ there was no difference in the proportion of vesicles marked by Lc3, but most vesicular bacteria are degraded by 26hpi (Figure $2 \mathrm{G}$ ), showing that a rapid Lc3 response to $S$. aureus infection occurs. In contrast, vesicles containing $S$. aureus are significantly more likely to have Lc3 puncta associated at 2hpi (Figure $2 \mathrm{H}$, Supplementary Figure 1D), although most bacteria are still cleared by $26 \mathrm{hpi}$ and there was no significant change in the association of Lc3 puncta to $S$. aureus in the cytosol over time (Figure 2I).

\section{Loss of p62 reduces zebrafish survival following Staphylococcus aureus infection}

We had demonstrated the steps of Lc3 and the autophagy receptor p62 recruitment in vivo in the degradation of $S$. aureus by neutrophils suggesting a function for p62 in immunity to $S$. aureus infection by targeting degradation of bacteria that escaped the phagosome. To test this prediction we examined the role of $\mathrm{p} 62$ in $S$. aureus zebrafish infection using a morpholinomodified antisense oligonucleotide (morpholino) targeting p62 (van der Vaart et al., 2014) to knock-down $p 62$ expression in the zebrafish larvae. Knock down of $p 62$ resulted in a significant reduction in zebrafish survival following $S$. aureus infection, compared to control larvae, supporting a requirement for p62 in the control of $S$. aureus infection (Figure 3A). Knockdown of $p 62$ did not reduce larval survival for heat-killed $S$. aureus or the non-virulent but closely related bacterium Micrococcus luteus (Supplemental Figure 4B, C), suggesting p62 is important for restriction of pathogenic bacteria that escapes the phagosome. To further support this conclusion, we generated a 062 mutant zebrafish (sh558) that lacked a functional UBD domain in p62, inhibiting the ability of p62 to bind to ubiquitinated cargo (Figure 3C). In agreement with our knock down study, the p62 mutant zebrafish (sh558) larvae were significantly more susceptible to $S$. aureus infection than wild-type control zebrafish (Figure 3B). Thus, in addition to demonstrating how Lc3 and p62 were localised during intracellular 
handling of $S$. aureus by neutrophils, we could independently show the requirement of p62 in the outcome of infection.

Both p62 morpholino and p62 mutant zebrafish (sh558) techniques do not block p62 function in neutrophils specifically, therefore we next aimed to determine whether the loss of p62 was important in neutrophils during $S$. aureus infection. Interestingly, there was no difference between the survival of our GFP-p62 reporter and wild-type controls (Supplementary Figure $4 A)$, suggesting that endogenous $p 62$ expression is sufficient for restriction of the small proportion of bacteria which reside in the cytosol. First, using TSA staining of 1dpi larvae to visualise neutrophils within p62 mutant (sh558) and control larvae, we found a non-significant $(p=0.1039)$ increase in neutrophils containing $S$. aureus (Figure 3D). A small effect was expected due to the small proportion of cytosolic bacteria which are likely targeted by $p 62$ during infection. It was therefore likely that showing a difference in the number of infected neutrophils would have required a very large number of infections; we were able to calculate that the observed differences would require a group size of 270 . Next, using p62 morphants and control larvae, a comparison of the number of bacteria present within neutrophils at $1 \mathrm{dpi}$ was completed in the $\mathrm{Tg}$ (mpx:eGFP)i114 larvae. In agreement with the p62-UBD mutant data, a non-significant $(p=0.115)$ increase of neutrophils containing $S$. aureus was observed in $p 62$ morphants in comparison to wild-type controls (Figure $3 \mathrm{E}$ ), again we were able to calculate that the observed differences would require a large group size of 219. However, examination of the bacterial location revealed a significant increase in the number of cytosolic $S$. aureus in the p62 morphants in comparison to control fish (Figure 3F), suggesting loss of p62 is important for the control of cytosolic $S$. aureus by neutrophils. Thus, we could show that loss of $p 62$ leads to an increase in bacterial burden within neutrophils and that p62 is likely targeting the small proportion of bacteria which escape to the cytosol.

\section{Discussion}

Using the unique attributes of long-term high-resolution imaging and genetic manipulation of zebrafish larvae we have shown the dynamics of Lc3 and p62 on the $S$. aureus containing vacuole, their relation to bacterial degradation, and how p62 recognises cytosolic bacteria meaning that loss of p62 activity is sufficient to increase mortality following $S$. aureus infection.

Loss of zebrafish p62, through morpholino-mediated knockdown, significantly increased susceptibility to infection to $S$. aureus. This is the first in vivo evidence that $p 62$ is important in the outcome of intracellular handling of $S$. aureus. To confirm the $p 62$ knockdown data, we generated a zebrafish p62 mutant lacking the UBD domain, which confirmed a significant increase in susceptibility to zebrafish in $S$. aureus infection. This suggests that for $S$. aureus 
infection control, the p62 UBD, which is able to bind to ubiquitinated $S$. aureus (Neumann et al., 2016; Singh et al., 2017), is important for host control of infection. In addition to its role as an autophagy receptor, p62 can aid killing of pathogens through delivery of anti-microbial peptides (Ponpuak et al., 2010); it is possible that anti-microbial peptides delivered by p62 are important in neutrophil control of $S$. aureus infection. The $p 62$ zebrafish mutant represents a valuable tool in the analysis of selective autophagy in infection, which may also be useful for study of other intracellular pathogens or in other diseases where autophagy is implicated in pathology, for example in neurodegenerative disorders.

Although in vitro studies have described co-localisation of p62 and autophagy in pathogen handling, until now, no evidence of direct p62 interactions with these pathogens has been shown in neutrophils or in vivo. Interaction of p62 with $S$. aureus has been demonstrated through in vitro studies using fibroblasts and epithelial cells (Neumann et al., 2016; Singh et al., 2017). In vitro data shows $S$. aureus can be targeted for autophagic degradation by p62 (Neumann et al., 2016; Singh et al., 2017), where puncta appear to be co-localised with $S$. aureus. Our new zebrafish GFP-p62 reporter shows cytosolic puncta formation, which has also been observed in other cell culture studies, both endogenous expression and using similar GFP-p62 reporter systems (Bjørkøy et al., 2005; Pankiv et al., 2007; Larsen et al., 2010). By comparing GFP-p62 puncta marking of intracellular $S$. aureus with the location of bacteria over time, it is interesting to note that p62 marking is reduced over time for cytosolic bacteria (which appear to be a small population that persist throughout infection); this may indicate that cytosolic bacteria marked with p62 are degraded. Furthermore, at later time points in S. aureus infection the number of GFP-p62 puncta is reduced within infected cells, suggesting when bacteria escape the phagosome, p62 becomes important in controlling cytosolic bacteria.

We show that most $S$. aureus is contained within a vesicle soon after infection and that by 26 hpi most $S$. aureus are absent from neutrophils. Of note, some images show bacteria outside neutrophils, these represent bacteria which have been phagocytosed by macrophages, which has previously been described (Prajsnar et al., 2008). The large reduction of neutrophils containing bacteria from $2 \mathrm{hpi}$ to $26 \mathrm{hpi}$, leaving a small population at $26 \mathrm{hpi}$, may be representative of a niche for bacterial persistence and/or proliferation. The role of neutrophils as an intracellular niche has previously been described to be important in determining the outcome of $S$. aureus infection (Thwaites and Gant, 2011; Prajsnar et al., 2012; Pollitt et al., 2018). Interestingly, it appears that Lc3 marks the majority of vesicles containing bacteria. Lc3 localisation to $S$. aureus may represent Lc3 recruitment to autophagosomes, however since recruitment is observed at early infection time points, it may represent Lc3-associated phagocytosis (LAP), which is also observed in Listeria 
301 degraded, it appears that Lc3 marking of vesicles could lead to bacterial degradation in the 302 zebrafish.

303 Thus, we demonstrate that host p62 is beneficial for the host outcome following $S$. aureus 304 infection and that $\mathrm{p} 62$ mediated control of cytosolic bacteria within neutrophils may represent 305 one of many mechanisms employed by the host in immunity to this versatile pathogen. 


\section{Ethics statement}

312 Animal work was carried out according to guidelines and legislation set out in UK law in the 313 Animals (Scientific Procedures) Act 1986, under Project License PPL 40/3574 or 314 P1A4A7A5E). Ethical approval was granted by the University of Sheffield Local Ethical 315 Review Panel. Animal work completed in Singapore was completed under the Institutional 316 Animal Care and Use Committee (IACUC) guidelines, under the A*STAR Biological Resource 317 Centre (BRC) approved IACUC Protocol \#140977.

\section{Zebrafish husbandry}

Zebrafish strains were maintained according to standard protocols (Nüsslein-Volhard and Dahm, 2002). For animals housed in the Bateson Centre aquaria at the University of Sheffield, adult fish were maintained on a $14: 10$-hour light/dark cycle at $28^{\circ} \mathrm{C}$ in UK Home Office approved facilities. For animals housed in IMCB, Singapore, adult fish were maintained on a $14: 10$-hour light/dark cycle at $28^{\circ} \mathrm{C}$ in the IMCB zebrafish facility. LWT and AB wild-type larvae were used in addition to transgenic lines, $T g(l y z: e G F P-p 62) i 330$ created in this study, $T g$ (lyz:RFP-GFP-Lc3)sh383 (Prajsnar et al., 2019), Tg(lyz:nfsB-mCherry)sh260 (Buchan et al., 2019) (these fish encode nitroreductase gene nsfB within neutrophils which allows ablation of cells following metronidazole treatment, which was not used in this study) and Tg(mpx:eGFP)i114 (Renshaw et al., 2006). Generation of p62 sh558 mutant zebrafish is described below. Larvae were maintained in $\mathrm{E} 3$ plus methylene blue at $28^{\circ} \mathrm{C}$ until $5 \mathrm{dpf}$.

\section{S. aureus culture}

The Staphylococcus aureus strain $\mathrm{SH} 1000$ was used in this study. A single bacterial colony was placed in $10 \mathrm{ml}$ brain heart infusion (BHI) medium (Oxoid number 1) overnight at $37^{\circ} \mathrm{C}$, $250 \mathrm{rpm} .500 \mu \mathrm{l}$ of this overnight culture was then added to $50 \mathrm{ml}$ of $\mathrm{BHI}$ medium and incubated at $37^{\circ} \mathrm{C}, 250 \mathrm{rpm}$ until $\mathrm{OD}_{600} 1$. The bacteria were then pelleted at $4500 \mathrm{rpm}, 4^{\circ} \mathrm{C}$ for 15 minutes. The bacteria were then re-suspended in PBS (Oxoid, BR0014G), using a volume to dilute to the required dose, with $1500 \mathrm{cfu} / \mathrm{nL}$ being standard. Bacteria were incubated on ice for a short period, until use. Strains used: SH1000 wild-type strain (Horsburgh et al., 2002), SH1000pMV158-mCherry (Boldock et al., 2018), SH1000-pMV158-GFP (Boldock et al., 2018).

\section{Zebrafish micro-injection}

340 For p62 morpholino micro-injections: Larvae were injected immediately after fertilisation using a p62 morpholino (van der Vaart et al., 2014). A standard control morpholino

342 (Genetools) was used as a negative control. For injection of S. aureus, zebrafish larvae were 
injected at $1 \mathrm{dpf}$ (for survival analysis, (Prajsnar et al., 2008)) or $2 \mathrm{dpf}$ (for microscopy analysis) and monitored until a maximum of $5 \mathrm{dpf}$. Larvae were anesthetised by immersion in $0.168 \mathrm{mg} / \mathrm{mL}$ tricaine in E3 and transferred onto $3 \%$ methyl cellulose in E3 for injection. For $S$. aureus $1 \mathrm{nl}$ of bacteria, containing $1500 \mathrm{cfu}$, was injected into the yolk sac circulation valley. Larvae were transferred to fresh E3 to recover from anaesthetic. Any zebrafish injured by the needle/micro-injection were removed from the procedure. Zebrafish were maintained at $28^{\circ} \mathrm{C}$.

\section{Generation of $T g(l y z: e G F P-p 62) i 330$ transgenic line}

The generation of the $T g(l y z: e G F P-p 62)$ i330 line was performed using the Gateway ${ }^{\mathrm{TM}}$ system in combination with Tol2 transgenesis (Tol2Kit) (Kwan et al., 2007). To make the required expression clone, pDest(lyz:eGFP-p62), the p5E-lyz entry clone (Elks et al., 2011) and the pME-eGFP-nostop middle entry vectors were used. The destination vector pDestTol2CG, was chosen which included Tol2 sites for integration into the genome, in addition to a GFP heart marker. The required p62 3' entry vector, and expression clone pDest(lyz:eGFP-p62) were constructed following the Multisite Gateway ${ }^{\mathrm{TM}}$ three-fragment vector construction kit. To generate Tol2 mRNA, a pT3Tol2 plasmid was used. The DNA pT3Tol2 plasmid was linearised through a restriction site digest. Tol2 mRNA was generated by a transcription reaction (Ambion T3 mMessage Machine) from the linear pT3Tol2 plasmid. Tol2 mRNA and pDest(lyz:eGFPp62) were co-injected into a single cell (at the single cell stage) of wild-type $A B$ larvae. $A 1 \mathrm{nl}$ injection contained 30pg of Tol2 mRNA and 60pg of pDest(lyz:eGFP-p62).

\section{Microscopy of infected zebrafish}

Larvae were anaesthetized $0.168 \mathrm{mg} / \mathrm{mL}$ tricaine in $\mathrm{E} 3$ and mounted in $0.8 \%$ low melting agarose onto glass bottom microwell dishes (MatTek P35G-1.5-14C). An UltraVIEW VoX spinning disk confocal microscope (Perkin Elmer, Cambridge, UK) was used for imaging neutrophils within larvae. 405nm, 445nm, 488nm, 514nm, 561nm and 640nm lasers were available for excitation. Most cellular level imaging was completed in the caudal hematopoietic tissue (CHT) using a 40x oil objective (UplanSApo 40x oil (NA 1.3)). In some cases a 20x objective was used for whole larvae imaging. GFP, TxRed emission filters were used and bright field images were acquired using a Hamamatsu C9100-50 EM-CCD camera. Volocity software was used. Between early and late time points zebrafish larvae were placed back into E3 and maintained at $28^{\circ} \mathrm{C}$.

\section{pHrodo staining of $S$. aureus}


Bacterial strains were prepared for injected (as above) and re-suspended into PBS pH 9. pHRODO (Thermofisher, P36600) was added at a ratio of $1: 200$ and incubated at $37^{\circ} \mathrm{C}$ for 30 minutes, shaking, in the dark. The bacteria were suspended in PBS pH 8 and washed through a series of solutions, (Tris pH8.5, PBS pH 8) and finally re-suspended into PBS $\mathrm{pH} 7.4$ for injection.

\section{Tyramide Signal Amplification (TSA) Staining}

Following $S$. aureus infection, larvae were fixed in $4 \%$ paraformaldehyde overnight at $4{ }^{\circ} \mathrm{C}$. Once fixed, larvae were washed in PBS thrice. Staining of neutrophils (specifically myeloperoxidase activity) in LWT wild-type larvae was completed using TSA staining kit (Cy5-TSA Cyanine Kit, PerkinElmer, NEL705A001KT). Fish were incubated in a 1:100 ratio of Cy5-TSA:amplification diluent at $28^{\circ} \mathrm{C}$ for 10 minutes in the dark. Larvae were washed thrice in PBS before imaging.

\section{$\underline{\text { TEM of infected zebrafish }}$}

Specimens were fixed in $2.5 \%$ Glutaldehyde/0.1M Sodium Cacodylate and postfixed $2 \%$ aqueous Osmium Tetroxide, dehydrated through a graded series of ethanol, and cleared in epoxypropane (EPP) and then infiltrated in 50/50 Araldite resin: EPP mixture overnight on a rotor. This mixture was replaced with two changes over 8 hours of fresh Araldite resin mixture before being embedded and cured in a 60 degrees $\mathrm{C}$ oven for $48-72$ hours. Ultrathin sections, approximately $85 \mathrm{~nm}$ thick, were cut on a Leica UC6 ultramicrotome onto 200 mesh copper grids. These were stained for 10 mins with saturated aqueous Uranyl Acetate followed by Reynold's Lead Citrate for 5 mins. Sections were examined using an FEI Tecnai Transmission Electron Microscope at an accelerating voltage of $80 \mathrm{Kv}$. Electron micrographs were recorded using Gatan Orius 1000 digital camera and Gatan Digital Micrograph software

\section{Image analysis}

Image analysis was performed using ImageJ software, to quantify the number of $S$. aureus cells within neutrophils, and to quantify GFP-p62 puncta and Lc3 co-localisation to these pathogens.

\section{Drug treatment of zebrafish}

Larvae were treated with autophagy inducers and inhibitors through immersion in E3 medium. All drugs were sourced from Sigma-Aldrich, UK. The Bay K8644 was added to the E3 to the 
405

406

407

408

409

410

411

412

413

414

415

416

417

418

419

420

421

422

423

424

425

426

427

428

429

430

431

432

433

434

435

required concentration, Bay-K $68441 \mu \mathrm{M}$. Larvae were incubated at $28^{\circ} \mathrm{C}$ for 24 hours before microscopy. Zebrafish were not anaesthetised for immersion drug treatments.

\section{Generation of $p 62$ mutant}

A zebrafish $p 62$ mutant was generated using CRISPR/Cas9 mutagenesis. A guide RNA targeting exon 8 of zebrafish p62 (ACAGAGACTCCACCAGCCTA) was inserted into a published oligonucleotide scaffold (Talbot and Amacher, 2014) and injected together with recombinant Cas9 protein (New England Biolabs) into 1-2 cell stage zebrafish (AB strain). Efficiency of mutagenesis was confirmed using high resolution melt curve analysis as previously described (Sutton et al., 2007) and several founders were identified. P62 ${ }^{\text {sh558 }}$ carries a 10 base pair deletion resulting in a frameshift and premature truncation of $p 62$ in the ubiquitin-associated (UBA) domain.

\section{$\underline{\text { Statistical analysis }}$}

Statistical analysis was performed as described in the results and figure legends. We used Graph Pad Prism 7 (v7.04) for statistical tests and plots.

\section{Acknowledgments:}

JFG was supported by an award from the Singapore A*STAR Research Attachment Programme (ARAP) in partnership with the University of Sheffield, and a Medical Research Council Grant (MR/R001111/1 with SAR and SJF). TKP was supported by an individual Marie Curie fellowship (PIEF-GA-2013-625975) and by AMR cross-council funding from the MRC to the SHIELD consortium "Optimising Innate Host Defence to Combat Antimicrobial Resistance" MRNO2995X/1. RDT and AJG were supported by the University of Sheffield. JJS was a Marie Curie fellow in the Initial Training Network FishForPharma (PITN-GA-2011- 289209). Work in the PWI lab was funded by the A*STAR Institute of Molecular and Cell Biology (IMCB) and the Lee Kong Chian School of Medicine. SAJ was supported by Medical Research Council and Department for International Development Career Development Award Fellowship MR/J009156/1 (http://www.mrc.ac.uk/). SAJ was additionally supported by a Krebs Institute Fellowship (http://krebsinstitute.group.shef.ac.uk/), and Medical Research Council Centre grant (G0700091). SAR was supported by a Medical Research Council Programme Grant (MR/M004864/1) (http://www.mrc.ac.uk/). Imaging was completed at the Wolfson Light Microscopy Facility. We thank aquarium staff at the Bateson Centre (Sheffield) and the IMCB (Singapore) for zebrafish husbandry. 


\section{Author contributions}

438 JFG, TKP, SAJ, AJG, PWI and SAR conceived this study and designed and interpreted 439 experiments. JFG prepared manuscript with important intellectual input from TKP, SAJ, AJG, 440 SJF and SAR. JFG and JJS conducted bacterial fate and location analysis. JFG performed 441 GFP-p62 reporter line generation and characterisation, zebrafish infection, morpholino 442 experiments, intracellular imaging and subsequent analysis. AKT performed zebrafish 443 infection. CJH performed TEM. SAJ performed imaging of bacteria degradation. TKP 444 performed p62 morpholino experiments. AJG and RDT generated the p62 stable mutant line. 
Amer, A. O. and Swanson, M. S. (2005) 'Autophagy is an immediate macrophage response to Legionella pneumophila', Cellular Microbiology, 7(6), pp. 765-778. doi: 10.1111/j.14625822.2005.00509.x.

450 Bayles, K. W. et al. (1998) 'Intracellular Staphylococcus aureus escapes the endosome and induces apoptosis in epithelial cells.', Infection and immunity, 66(1), pp. 336-42.

Bjørkøy, G. et al. (2005) 'p62/SQSTM1 forms protein aggregates degraded by autophagy and has a protective effect on huntingtin-induced cell death', The Journal of Cell Biology, 454 171(4).

Bjørkøy, G. et al. (2009) 'Chapter 12 Monitoring Autophagic Degradation of p62/SQSTM1', in, pp. 181-197. doi: 10.1016/S0076-6879(08)03612-4.

Boldock, E. et al. (2018) 'Human skin commensals augment Staphylococcus aureus pathogenesis', Nature Microbiology, 3, pp. 881-890. doi: 10.1038/s41564-018-0198-3.

Buchan, K. D. et al. (2019) 'A transgenic zebrafish line for in vivo visualisation of neutrophil myeloperoxidase', bioRxiv. Cold Spring Harbor Laboratory, p. 456541. doi: 10.1101/456541.

Deretic, V. and Levine, B. (2009) 'Autophagy, immunity, and microbial adaptations', Cell host \& microbe, 5(6), pp. 527-549. doi: 10.1016/j.chom.2009.05.016.Autophagy.

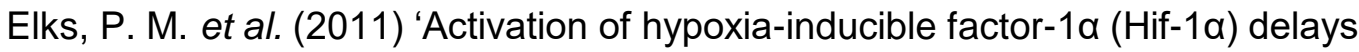
inflammation resolution by reducing neutrophil apoptosis and reverse migration in a zebrafish inflammation model.', Blood, 118(3), pp. 712-22. doi: 10.1182/blood-2010-12324186.

Farré, J.-C. and Subramani, S. (2016) 'Mechanistic insights into selective autophagy pathways: lessons from yeast', Nature Reviews Molecular Cell Biology, 17(9), pp. 537-552. doi: 10.1038/nrm.2016.74.

Gatica, D., Lahiri, V. and Klionsky, D. J. (2018) 'Cargo recognition and degradation by selective autophagy.', Nature cell biology. NIH Public Access, 20(3), pp. 233-242. doi: 10.1038/s41556-018-0037-z.

Gluschko, A. et al. (2018) 'The $\beta 2$ Integrin Mac-1 Induces Protective LC3-Associated Phagocytosis of Listeria monocytogenes.', Cell host \& microbe. Elsevier, 23(3), pp. 324337.e5. doi: 10.1016/j.chom.2018.01.018.

Gresham, H. D. et al. (2000) 'Survival of Staphylococcus aureus inside neutrophils 22.

Gutierrez, M. G. et al. (2005) 'Autophagy induction favours the generation and maturation of the Coxiella-replicative vacuoles', Cellular Microbiology. Blackwell Science Ltd, 7(7), pp. 981-993. doi: 10.1111/j.1462-5822.2005.00527.x.

Hall, C. et al. (2007) 'The zebrafish lysozyme C promoter drives myeloid-specific expression 10.1186/1471-213X-7-42. 10.1083/jcb.200309161. 
Staphylococcus aureus 8325-4', Journal of bacteriology, 184(19), pp. 5457-5467. doi: 10.1128/JB.184.19.5457.

Kwan, K. M. et al. (2007) 'The Tol2kit: A multisite gateway-based construction kit forTol2 transposon transgenesis constructs', Developmental Dynamics, 236(11), pp. 3088-3099. doi: $10.1002 /$ dvdy.21343.

Larsen, K. B. et al. (2010) 'A reporter cell system to monitor autophagy based on p62/SQSTM1', Autophagy, 66, pp. 784-793.

Levine, B., Mizushima, N. and Virgin, H. (2011) 'Autophagy in immunity and inflammation', Nature, 469(7330), pp. 323-335. doi: 10.1038/nature09782.Autophagy.

Mizushima, N. et al. (2008) 'Autophagy fights disease through cellular self-digestion', Nature, 451(7182), pp. 1069-1075. doi: 10.1038/nature06639.Autophagy.

Mostowy, S. et al. (2011) 'p62 and NDP52 proteins target intracytosolic Shigella and Listeria to different autophagy pathways.', The Journal of biological chemistry. American Society for Biochemistry and Molecular Biology, 286(30), pp. 26987-95. doi: 10.1074/jbc.M111.223610.

Neumann, Y. et al. (2016) 'Intracellular Staphylococcus aureus eludes selective autophagy by activating a host cell kinase', Autophagy. Taylor \& Francis, 12(11), pp. 2069-2084. doi: 10.1080/15548627.2016.1226732.

Nüsslein-Volhard, C. (Christiane) and Dahm, R. (2002) Zebrafish : a practical approach. Oxford University Press.

Pankiv, S. et al. (2007) 'p62/SQSTM1 binds directly to Atg8/LC3 to facilitate degradation of ubiquitinated protein aggregates by autophagy.', The Journal of biological chemistry. American Society for Biochemistry and Molecular Biology, 282(33), pp. 24131-45. doi: 10.1074/jbc.M702824200.

Pollitt, E. J. G. et al. (2018) 'Staphylococcus aureus infection dynamics.', PLoS pathogens. Public Library of Science, 14(6), p. e1007112. doi: 10.1371/journal.ppat.1007112.

Ponpuak, M. et al. (2010) 'Delivery of cytosolic components by autophagic adaptor protein p62 endows autophagosomes with unique antimicrobial properties.', Immunity. NIH Public Access, 32(3), pp. 329-41. doi: 10.1016/j.immuni.2010.02.009.

Popovic, D. and Dikic, I. (2012) 'The molecular basis of selective autophagy', Biochemist, 34(2), pp. 24-30.

Prajsnar, T. K. et al. (2008) 'A novel vertebrate model of Staphylococcus aureus infection reveals phagocyte-dependent resistance of zebrafish to non-host specialized pathogens.', Cellular microbiology, 10(11), pp. 2312-25. doi: 10.1111/j.1462-5822.2008.01213.x.

Prajsnar, T. K. et al. (2012) 'A privileged intraphagocyte niche is responsible for disseminated infection of Staphylococcus aureus in a zebrafish model.', Cellular microbiology, 14(10), pp. 1600-19. doi: 10.1111/j.1462-5822.2012.01826.x.

Prajsnar, T. K. et al. (2019) 'The autophagic response to Staphylococcus aureus provides an intracellular niche in neutrophils', bioRxiv. Cold Spring Harbor Laboratory, p. 581223. doi: $10.1101 / 581223$.

Renshaw, S. A. et al. (2006) 'A transgenic zebrafish model of neutrophilic inflammation', Blood, 108(13).

Rogov, V. et al. (2014) 'Interactions between autophagy receptors and ubiquitin-like proteins form the molecular basis for selective autophagy.', Molecular cell, 53(2), pp. 167-78. doi: 10.1016/j.molcel.2013.12.014. 
Schnaith, A. et al. (2007) 'Staphylococcus aureus subvert autophagy for induction of caspase-independent host cell death.', The Journal of biological chemistry, 282(4), pp. 2695-706. doi: 10.1074/jbc.M609784200.

Sharma, V. et al. (2018) 'Selective Autophagy and Xenophagy in Infection and Disease.',

538 Frontiers in cell and developmental biology. Frontiers Media SA, 6, p. 147. doi:

$53910.3389 /$ fcell.2018.00147.

540 Singh, A. et al. (2017) 'TBK1 regulates p62/sqstm1 mediated autophagic clearance of 541 intracellular ubiquitinated Staphylococcus aureus in human epithelial cells', Translational 542 genetics and genomics, [online fi.

543 Sutton, B. C. et al. (2007) 'Detection of the JAK2V617F mutation by asymmetric PCR and melt curve analysis', Cancer Biomarkers. IOS Press, 3(6), pp. 315-324. doi: 10.3233/CBM2007-3605.

546 Talbot, J. C. and Amacher, S. L. (2014) 'A streamlined CRISPR pipeline to reliably generate 547 zebrafish frameshifting alleles.', Zebrafish. Mary Ann Liebert, Inc., 11(6), pp. 583-5. doi: 548 10.1089/zeb.2014.1047.

549 Tanida, I. (2011) 'Autophagy basics.', Microbiology and immunology, 55(1), pp. 1-11. doi: 550 10.1111/j.1348-0421.2010.00271.x.

551 Thwaites, G. E. and Gant, V. (2011) 'Are bloodstream leukocytes Trojan Horses for the 552 metastasis of Staphylococcus aureus?', Nature Reviews Microbiology, 9(3), pp. 215-222.

553 doi: $10.1038 /$ nrmicro2508.

554 van der Vaart, M. et al. (2014) 'The DNA Damage-Regulated Autophagy Modulator DRAM1 555 Links Mycobacterial Recognition via TLR-MYD88 to Autophagic Defense', Cell Host \& 556 Microbe, 15(6), pp. 753-767. doi: 10.1016/j.chom.2014.05.005.

557 Williams, A. et al. (2008) 'Novel targets for Huntington's disease in an mTOR-independent 558 autophagy pathway.', Nature chemical biology. Europe PMC Funders, 4(5), pp. 295-305.

559 doi: 10.1038/nchembio.79.

560 Yang, C.-T. et al. (2012) 'Neutrophils exert protection in the early tuberculous granuloma by 561 oxidative killing of mycobacteria phagocytosed from infected macrophages.', Cell host \& microbe. NIH Public Access, 12(3), pp. 301-12. doi: 10.1016/j.chom.2012.07.009.

Zhang, R. et al. (2019) 'The selective autophagy receptors Optineurin and p62 are both required for zebrafish host resistance to mycobacterial infection', PLOS Pathogens. Edited by M. A. Behr. Public Library of Science, 15(2), p. e1007329. doi: 10.1371/journal.ppat.1007329. 
Figure 1: Staphylococcus aureus location within neutrophils changes from vesicular to cytosolic throughout infection

A-C Tg(mpx:eGFP)i114 larvae were injected at 1dpf with 1500cfu SH1000-mCherry S. aureus, and imaged at early (1-5hpi) and late (24-28hpi) time points A Number of bacteria contained in neutrophils, with maximum 100 bacterial cells counted, (whole larvae imaged, $n=11-13$, Mann-Whitney test, ${ }^{* * \star *} p<0.0001,+/-S D$ ) B Proportion of neutrophils containing bacteria (whole larvae imaged, $n=11-12$, unpaired t-test, ${ }^{* * *}$ p $<0.0001,+/$ - SEM) C Tg(mpx:eGFP)i114 larvae were injected at $1 \mathrm{dpf}$ with $1500 \mathrm{cfu}$ SH1000-mCherry S. aureus, and imaged at 3 hours post infection. Images were captured every 5 mins for 12 hours at multiple $z$ planes to follow infected neutrophils over time. Scale bar is $5 \mu \mathrm{m}$ D-G $T g$ (lyz:RFP-GFP-LC3)sh383 larvae were injected at 2dpf with GFP $S$. aureus, and imaged in the CHT at $2 \mathrm{hpi}$, and $\sim 26 \mathrm{hpi}$. D The proportion of infected or non-infected neutrophils at $2 \mathrm{hpi}$ and $26 \mathrm{hpi}$ $\left({ }^{* * * *} p<0.0001\right.$ Chi Square test, $n=3,172 \mathrm{hpi}$ larvae, $1126 \mathrm{hpi}$ larvae) E S. aureus with Lc3 marking the entire vesicle, scale 9um, demonstrating a vesicle F S. aureus in the cytosol, scale $9 \mu \mathrm{m}$ G Proportion $S$. aureus events observed within vesicles or cytosol at $2 \mathrm{hpi}$ and $26 \mathrm{hpi}\left({ }^{* * *} \mathrm{p}<0.001\right.$, Fisher's exact test, $\mathrm{n}=3,17$ larvae at $2 \mathrm{hpi}$, and 11 larvae at 26hpi).

Figure 2: In vivo recruitment of GFP-p62 puncta during S. aureus infection

A Representative image of $S$. aureus observed within a likely "vesicle" with GFP-p62 puncta localisation, scale 7um B representative image of $S$. aureus observed within the cytosol with GFP-p62 puncta localisation, scale 9um C S. aureus within vesicles, co-localised with GFP-p62 at 2hpi and 26pi (CHT imaged, ns, Fisher's exact test, n=3, 14 larvae at $2 \mathrm{hpi}$, and 12 larvae at 26hpi) D S. aureus in the cytosol, co-localised with GFP-p62 at 2hpi and 26hpi (CHT imaged, ${ }^{*} p<0.05$, Fisher's exact test, $n=3$, 14 larvae at $2 \mathrm{hpi}$, and 12 larvae at 26hpi) E GFP-p62 puncta in the cytosol of infected and non-infected at 2hpi (CHT imaged, ns, Mann-Whitney test, $\mathrm{n}=3$, error bars +/- SD, 14 larvae) F GFP-p62 puncta in the cytosol of infected and non-infected at $26 \mathrm{hpi}\left(\mathrm{CHT}\right.$ imaged, ${ }^{* *} \mathrm{p}<0.01$, Mann-Whitney test, $\mathrm{n}=3$, error bars +/- SD, 12 larvae) G-I 2500cfu of GFP S. aureus injected into Tg(lyzC:RFP-GFP-Lc3)sh383, larvae imaged in the $\mathrm{CHT}$ at $2 \mathrm{hpi}(2 \mathrm{hpi})$ and $26 \mathrm{hpi}$ (26hpi). G Lc3 association to the entire $S$. aureus vesicle at $2 \mathrm{hpi}$ and $26 \mathrm{hpi}$ (ns, Fisher's test, $\mathrm{n}=3,172 \mathrm{hpi}$ larvae, $1126 \mathrm{hpi}$ larvae) $\mathbf{H}$ The number of $S$. aureus vesicles with Lc3 puncta ( ${ }^{*} p<0.05$, Fisher's test, $n=3,172 \mathrm{hpi}$ larvae, $1126 \mathrm{hpi}$ larvae) I The number of S. aureus events in the cytosol with Lc3 puncta at $2 \mathrm{hpi}$ and $26 \mathrm{hpi}$ (ns, Fisher's test, $n=3,172 \mathrm{hpi}$ larvae, 11 26hpi larvae).

Figure 3: Zebrafish survival is reduced following infection with Staphylococcus aureus in the absence of $\mathrm{p} 62$

A-B Zebrafish survival following $S$. aureus infection, larvae were injected with $1500 \mathrm{cfu}$ of $\mathrm{SH} 1000$ at 30hpf. A $p 62$ morphants or control morphants survival ( $n=3,74-80$ larvae per group, $p=0.004$, Log-rank, Mantel-Cox test) B $p 62$ mutant or wild-type sibling survival $(n=3,57-60$ larvae per group, $p=0.0168$, Log-rank, Mantel-Cox test) C Electropherograms showing the sequence of wild type and sh558 mutant p62. Dashed vertical lines show the location of the $5 \mathrm{bp}$ deletion. The position of the frameshift in the p62 protein is illustrated. Since this frameshift is located in the final coding exon we predict translation of a truncated p62 protein lacking the UBD domain. D-E Number of infected neutrophils at 26hpi following S. aureus infection, larvae were injected with 1500 cfu of SH1000 mCherry, imaging completed in $\mathrm{CHT}(\mathrm{D})$ or GFP $(\mathrm{E}, \mathrm{F})$ at 30hpf $\mathbf{D}$ p62 mutant or wild-type sibling ( $\mathrm{n}=3,19-36$ larvae per group, $\mathrm{p}=0.0168, \mathrm{p}=0.1039$, Mann-Whitney test, error bars $+/$ - SEM) $\mathrm{E}$ 62 morphants or control morphants in Tg(mpx:eGFP)i114 larvae $(n=3,32-34$ larvae per group, $p=0.115$, Mann-Whitney test, error bars $+/-$ SEM) F p62 morphants or control morphants $\operatorname{Tg}(m p x: e G F P) i 114$ larvae $(n=3,32-34$ larvae per group, ${ }^{* *} p<0.01$, Mann-Whitney test, error bars $+/$ - SEM) 
Supplementary Figure legends

\section{Supplementary Figure 1: Neutrophil interactions with S. aureus in the zebrafish model}

A $T g(m p x: e G F P) i 114$ larvae were injected at 1dpf with 1500cfu SH1000-mCherry S. aureus, and imaged at 3 hours post infection. Images were captured every 5 mins for 12 hours at multiple z planes to follow infected neutrophils over time. Scale bar is $5 \mu \mathrm{m}$. B Neutrophil counts in CHT region in 2dpf fish and 3dpf used for analysis ( $\mathrm{n}=6,29-45$ larvae per time point, no significant difference, unpaired Ttest) D-E Tg(lyz:RFP-GFP-Lc3)sh383 larvae were injected at 2dpf with GFP S. aureus, and imaged at $2 \mathrm{hpi}$, and $\sim 26 \mathrm{hpi}$. C S. aureus without Lc3 marking the entire vesicle, scale 5.8um. Also demonstrating a clear vesicle structure D $S$. aureus with Lc3 puncta marking the edge of a vesicle, (indicated with an arrowhead), scale $9 \mu \mathrm{m}$

\section{Supplementary Figure 2: The GFP-p62 reporter line expresses a GFP-p62 fusion protein within neutrophils}

A Illustration of the plasmid created to make the GFP-p62 zebrafish reporter line, pDest(lyz:eGFP-p62). B Representative image of neutrophils within larva resulting from crossing $T g$ (lyz:nfsB-mCherry)sh260 (which has mCherry fluorescent neutrophils), with $T g(l y z: e G F P-p 62) i 330$ which shows GFP expression within neutrophils. Image taken of the caudal haematopoietic tissue (CHT) shown in red box of diagram of larvae. C The percentage of neutrophils which express fluorescence of GFP alone, mCherry alone, or both GFP and mCherry using six representative larvae from a cross of Tg(lyz:nfsB-mCherry)sh260 with Tg(lyz:eGFP-p62)i330 zebrafish lines (CHT imaged, one-way ANOVA, Tukey's multiple comparisons ${ }^{* * * *}=p<0.001$ ). D High magnification imaging of an individual neutrophil from the CHT showing GFP puncta, but not mCherry puncta, from a Tg(lyz:nfsB-mCherry)sh260 cross with Tg(lyz:eGFP-p62)i330. E-F Quantification of images taken of Tg(lyz:eGFP-p62)i330 larvae in the CHT, treated with autophagy blocking drug Bay K8644. E Percentage of neutrophils with puncta present (Chisquare test, ${ }^{* *} p<0.01, n=3,15-20$ larvae per group) $\mathbf{F}$ Number of puncta observed within individual neutrophils (Error bars $+/-$ SD, Unpaired T-test ${ }^{* *} p<0.01, n=3,15-20$ larvae per group)

\section{Supplementary Figure 3: The intracellular location of Staphylococcus aureus within neutrophils changes throughout infection}

A-C and F-G Tg(lyz:eGFP-p62)i330 larvae were injected with mCherry S. aureus at 2dpf, and imaged at $2 \mathrm{hpi}$, and $\sim 26 \mathrm{hpi}$ in the $\mathrm{CHT}$. A The number of $S$. aureus events observed within neutrophils at $2 \mathrm{hpi}$ and $26 \mathrm{hpi}\left(\mathrm{n}=3,27\right.$ larvae at $2 \mathrm{hpi}$, and 18 larvae at $26 \mathrm{hpi}$ unpaired t-test, ${ }^{* *} \mathrm{p}<0.01,+/-$ SEM) $\mathbf{B}$ Proportion of neutrophils infected or non-infected with $S$. aureus at $2 \mathrm{hpi}$ and $26 \mathrm{hpi}$ ( ${ }^{* * * *} \mathrm{p}<0.001$, Fisher's exact test, $\mathrm{n}=3,14$ larvae at $2 \mathrm{hpi}$, and 12 larvae at 26hpi) $C$ Representative image of $S$. aureus observed within a likely "vesicle" D LWT zebrafish larvae injected with 1500cfu of SH1000 S. aureus and fixed at $\sim 18 \mathrm{hpi}$. Transmission electron micrograph of an infected neutrophil. Highlighted box shows zoom in of the vesicle contain S. aureus. E Tg(mpx:eGFP)i114 larvae injected at 1dpf with 1500cfu SH1000 S. aureus stained with pHrodo and imaged at $2 \mathrm{hpi}$ imaged in the CHT F representative image of $S$. aureus observed within the cytosol G Proportion S. aureus events observed within vesicles or cytosol at $2 \mathrm{hpi}$ and $26 \mathrm{hpi}{ }^{* * * *} \mathrm{p}<0.001$, Fisher's exact test, $n=3,14$ larvae at $2 \mathrm{hpi}$, and 12 larvae at $\left.26 \mathrm{hpi}\right)$

\section{Supplementary Figure 4: The role of p62 in zebrafish survival following infection}

A Zebrafish survival following $S$. aureus infection,GFP-p62 larvae or controls were injected with 1500cfu of SH1000 at 30hpf ( $n=3,39-44$ larvae per group, Log-rank, Mantel-Cox test) B Zebrafish survival following injection of heat-killed S. aureus (equivalent of 1500cfu SH1000) at $30 \mathrm{hpf}$, p62 morphants or control morphants ( $n=3,38-44$ larvae per group, Log-rank, Mantel-Cox test) C Zebrafish survival following injection of $M$. luteus 2000cfu at 30hpf, p62 morphants or control morphants ( $n=3,44-45$ larvae per group, Log-rank, Mantel-Cox test) 
668 Supplementary Video 1: GFP-p62 puncta co-localise with a vesicle containing S. aureus

669 Tg(lyz:eGFP-p62)i330 larvae were injected with 1500cfu mCherry S. aureus at 2dpf and imaged at 3hpi 670 in the CHT. Images were collected as fast as possible ( every 0.8 seconds) for up to 3 minutes. White 671 arrow indicates the location of the vesicle marked with GFP-p62 puncta.

\section{Supplementary Video 2: GFP-p62 puncta co-localise with S. aureus located in the cytosol}

$675 T g(l y z: e G F P-p 62)$ i330 larvae were injected with 1500cfu mCherry S. aureus at 2dpf and imaged at 3hpi 676 in the CHT. Images were collected as fast as possible ( every 0.8 seconds) for up to 3 minutes. White 677 arrow indicates the location of the S. aureus in the cytosol marked with GFP-p62 puncta. 

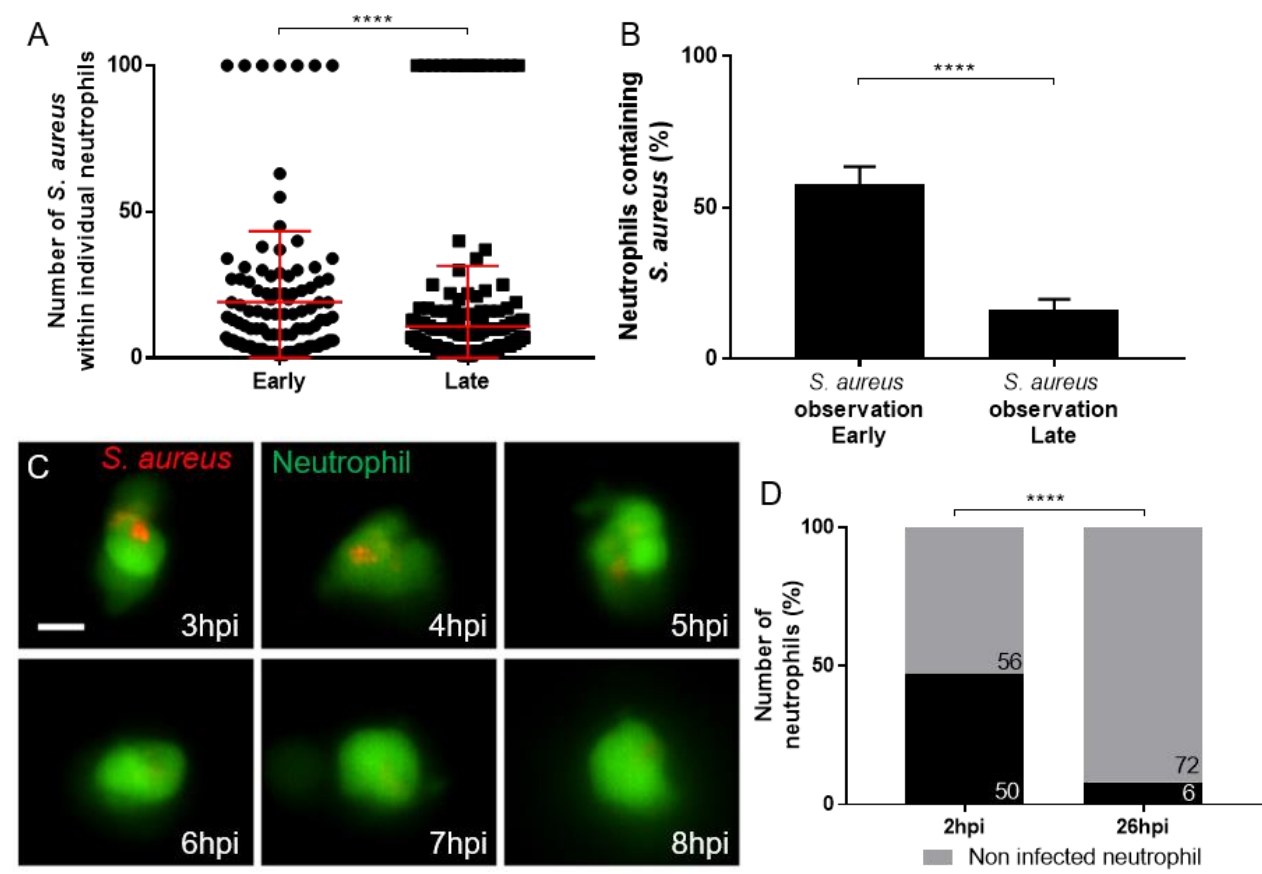

Chpi

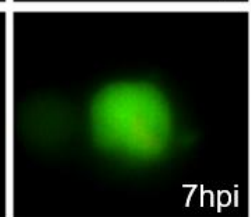

- Non infected neutroph
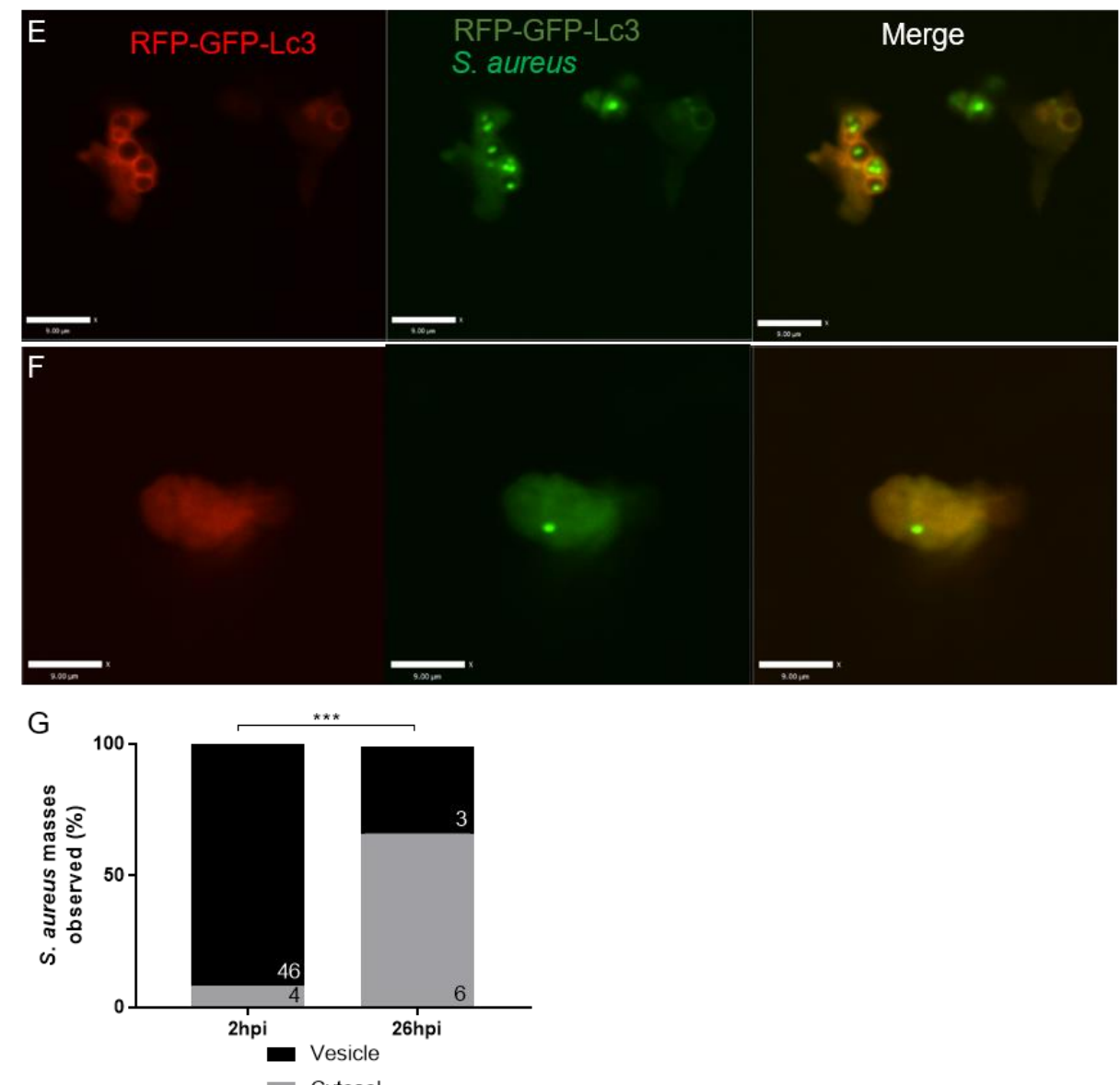

Figure 1: Staphylococcus aureus location within neutrophils changes from vesicular to 

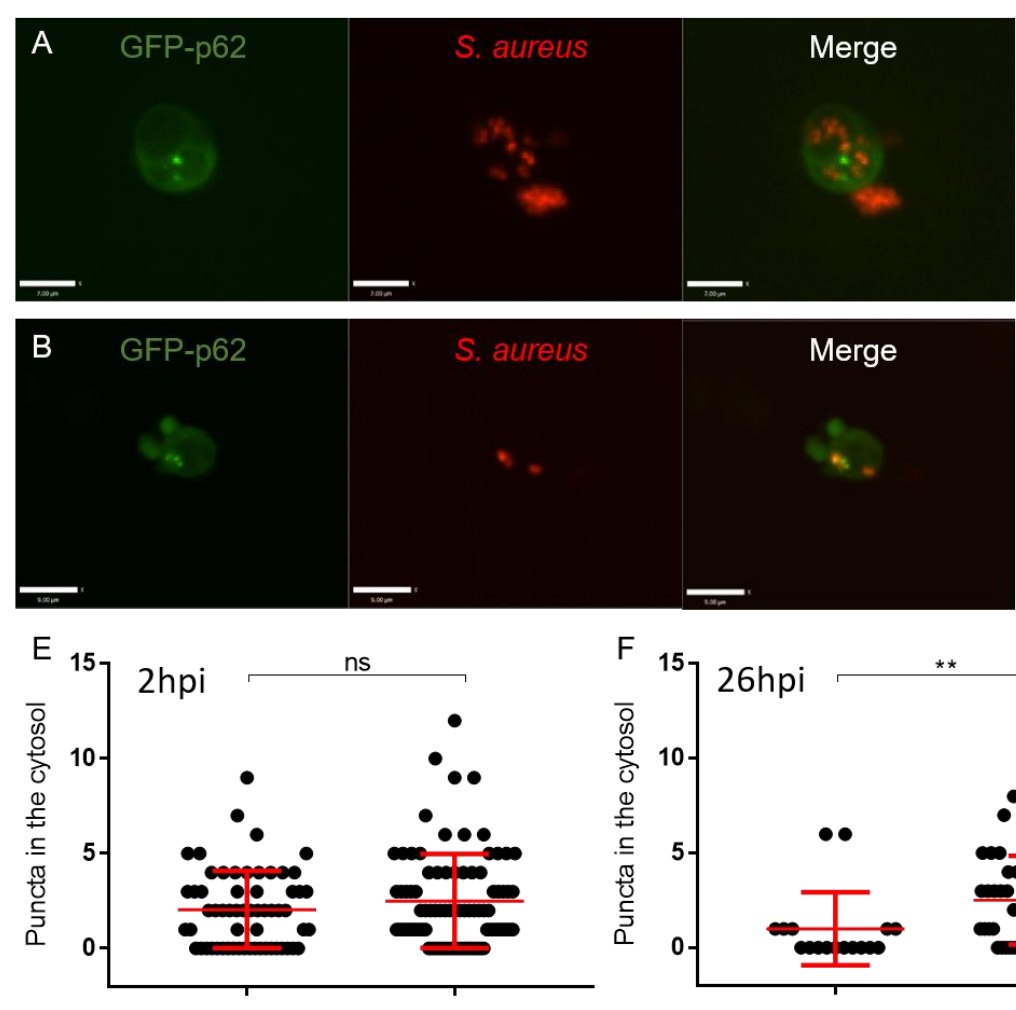

Infected Non-infected neutrophil neutrophil

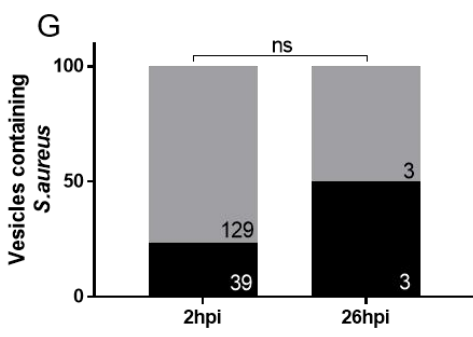

LC3 positive vesicle

- LC3 negative vesicle

neutrophil

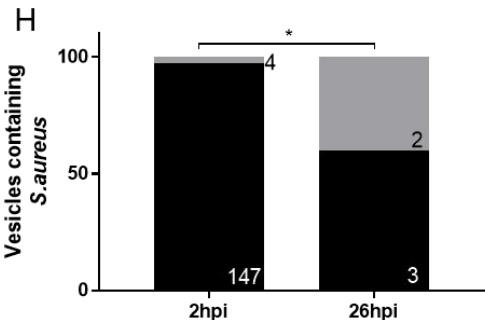

Lc3 puncta

- No Lc3 puncta

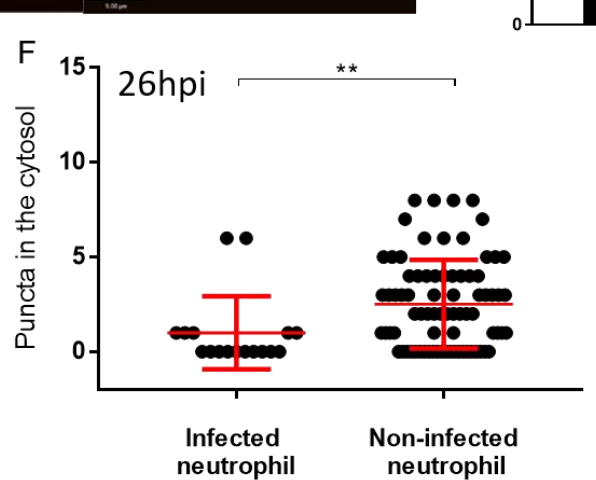

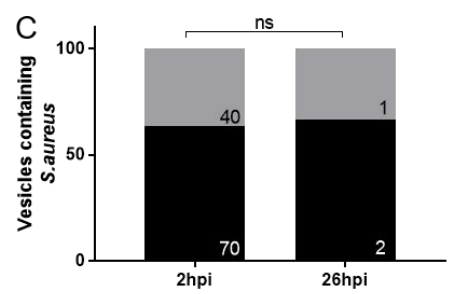

With 62 puncta

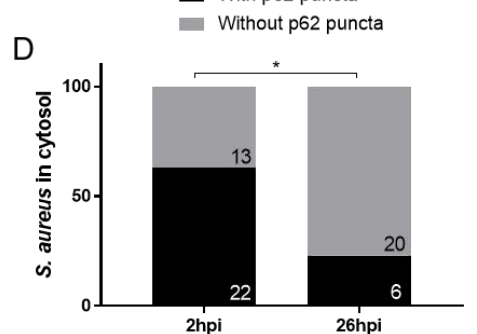

- With p62 puncta

Without p62 puncta

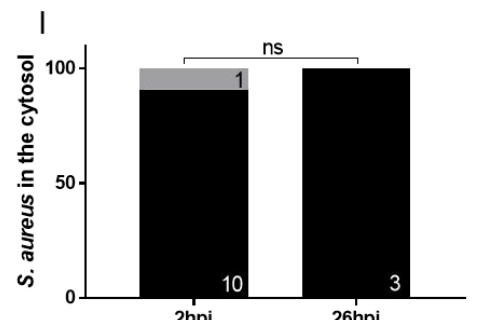

Lc3 puncta

- No Lc3 puncta 
A

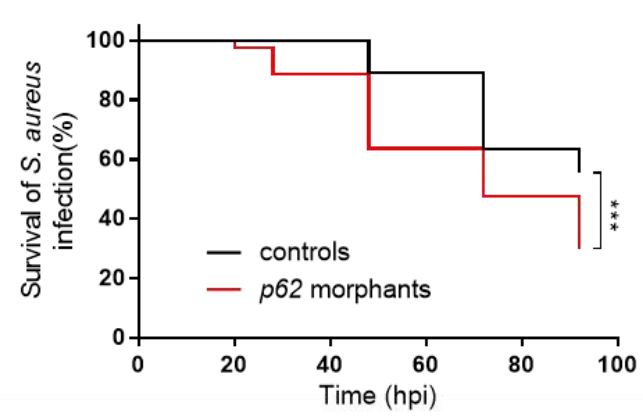

C

1

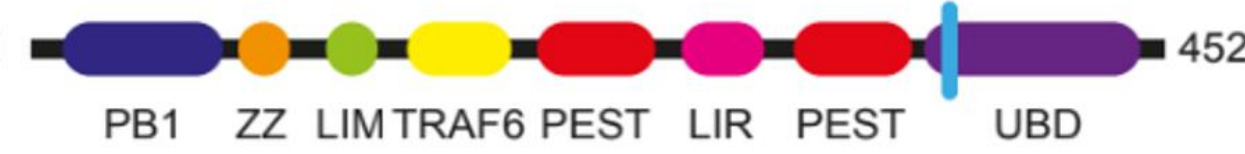

Wild Type

$\Delta 5 \mathrm{bp}$

D

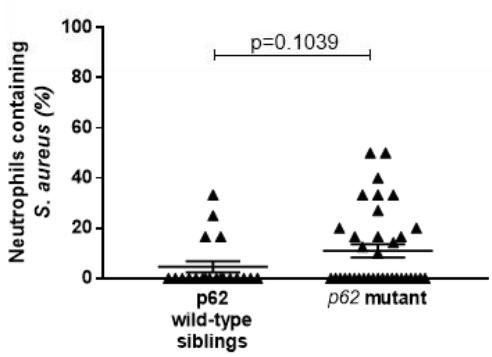

$\mathrm{E}$

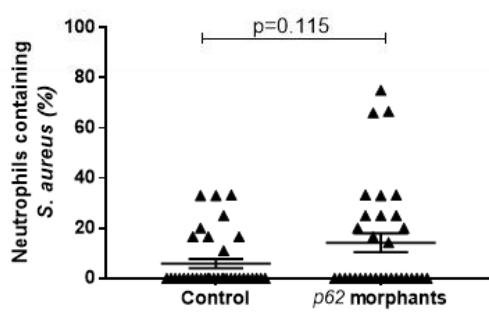

$\mathrm{F}$

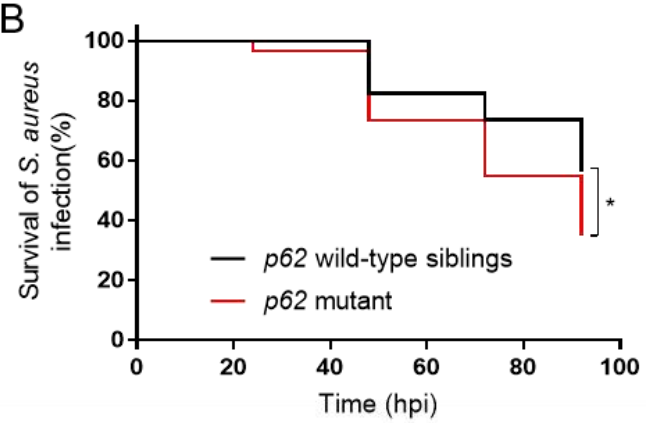

698

Figure 3: Loss of functional p62 reduces zebrafish survival following infection with

701

702 


\section{Supplementary figures}

706
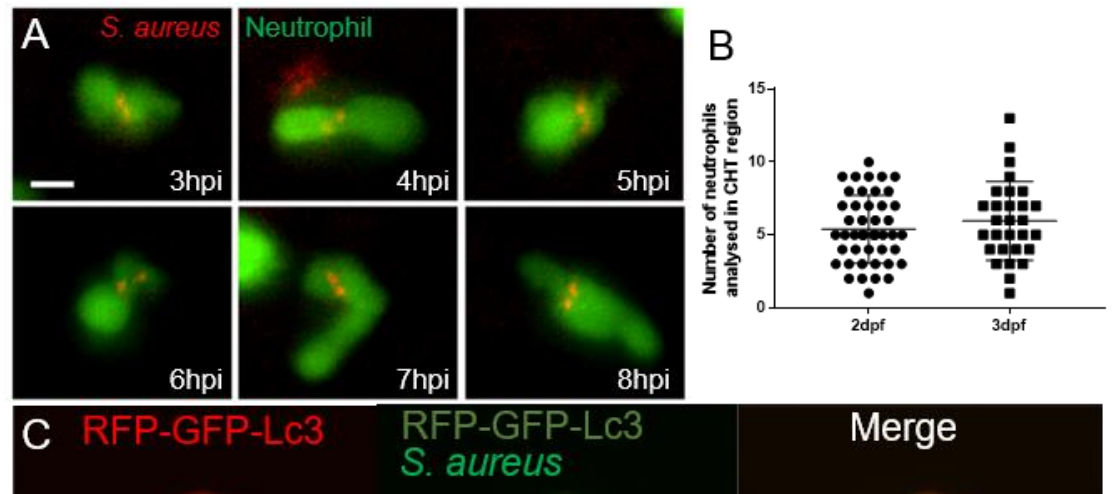

S. aureus

\section{Merge}

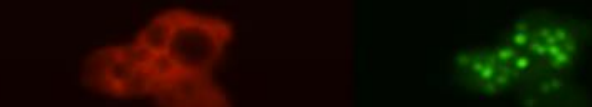

$\mathrm{D}$
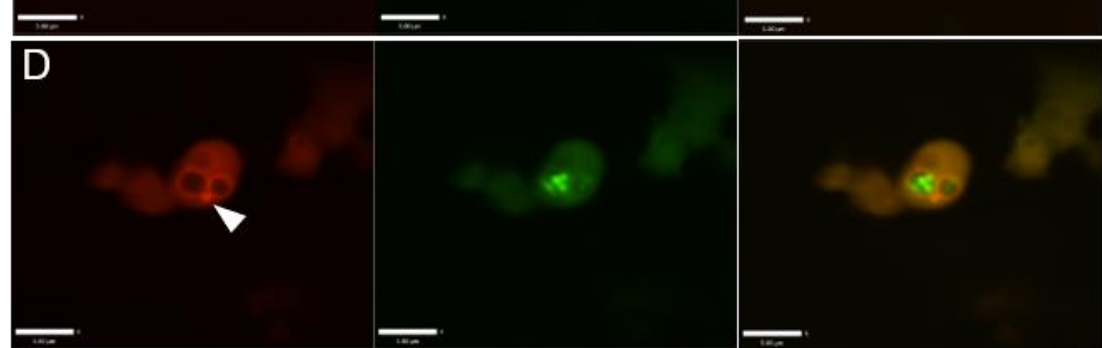

Supplementary Figure 1: Neutrophil interactions with $S$. aureus in the zebrafish 

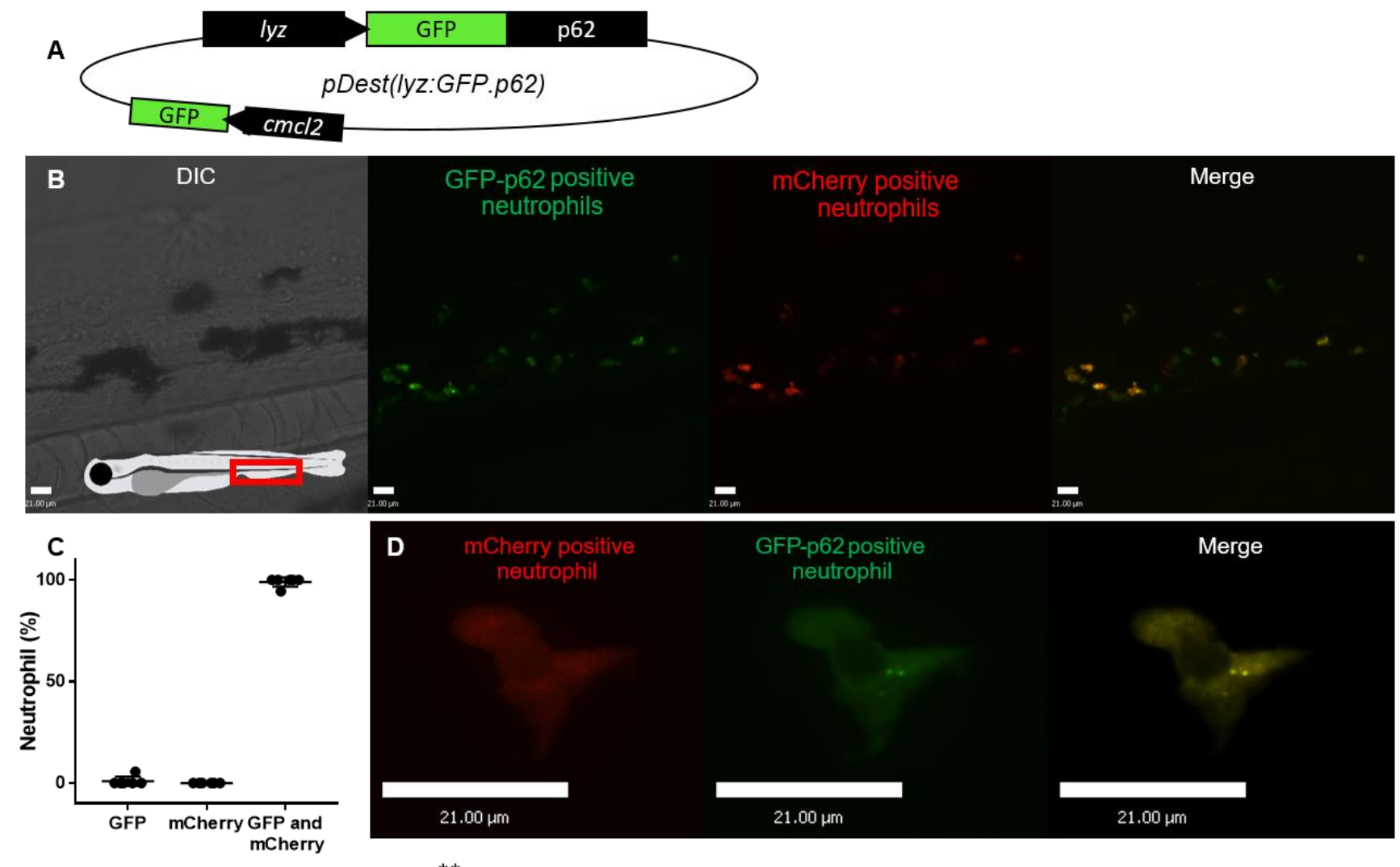

Merge

E

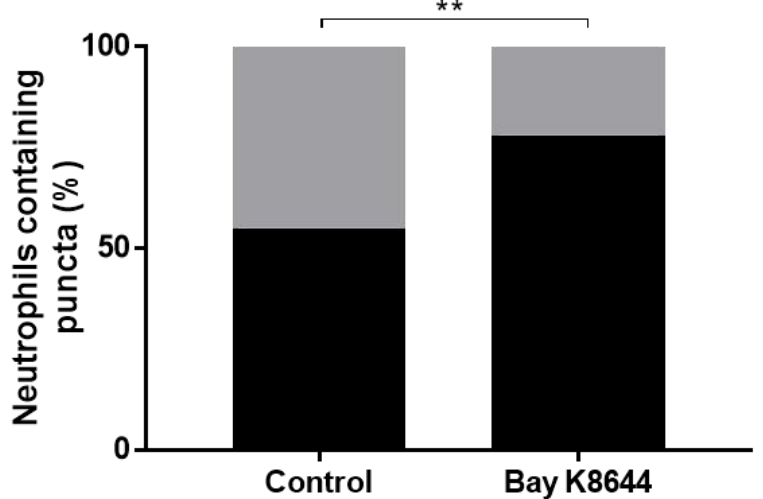

No puncta

- Puncta present

Supplementary Figure 2: The GFP-p62 reporter line expresses a GFP-p62 fusion protein within neutrophils

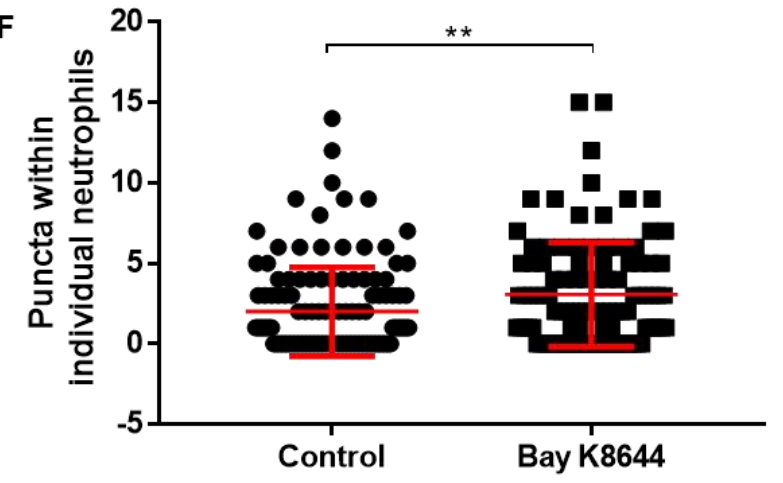




$$
\text { A }
$$
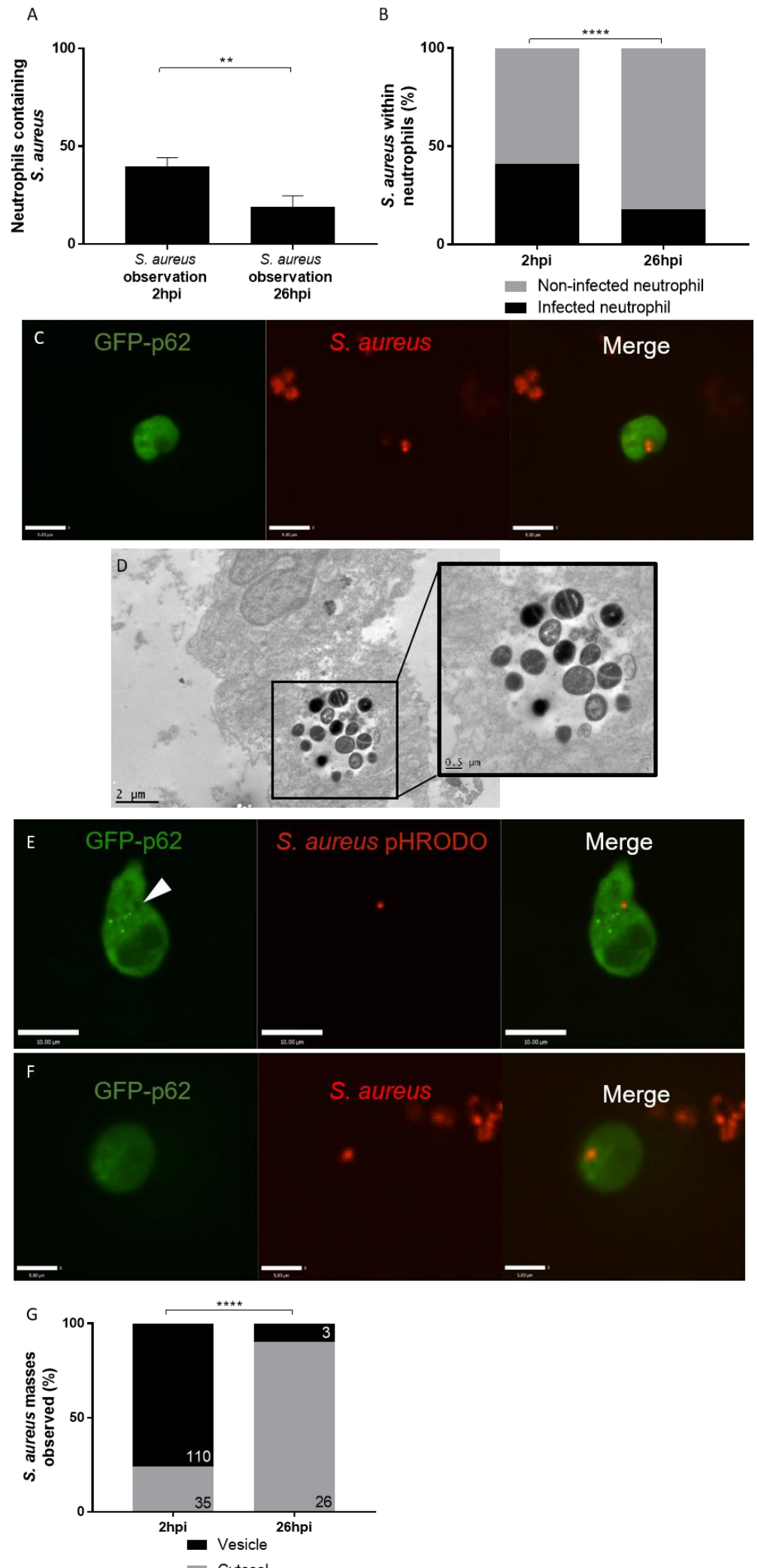

Cytosol 
A

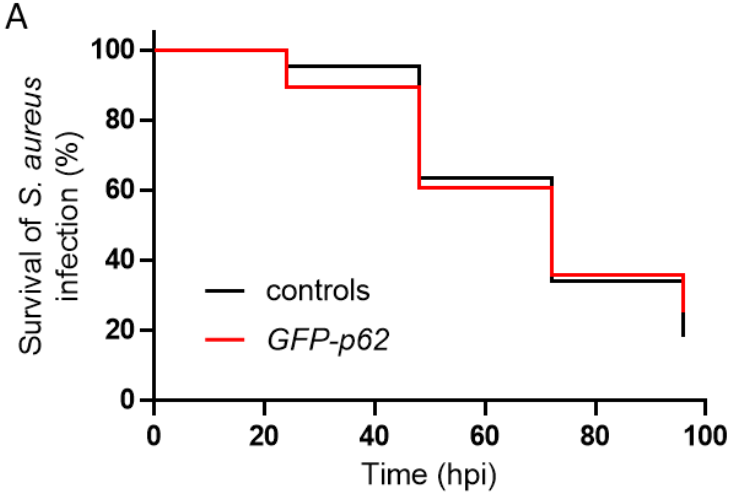

B

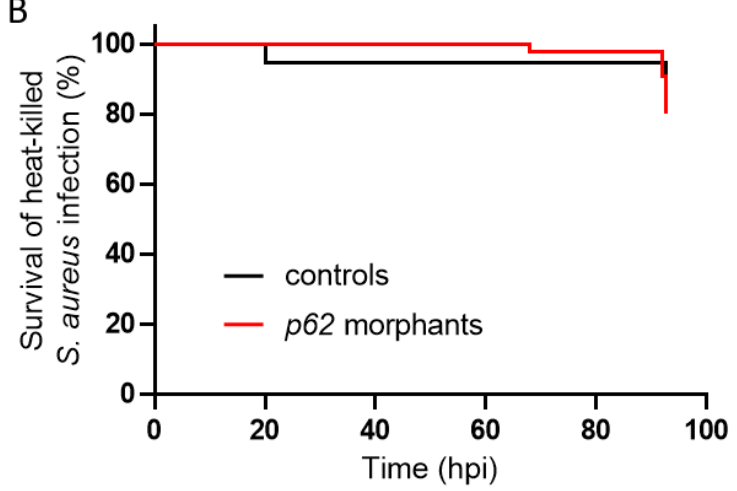

C

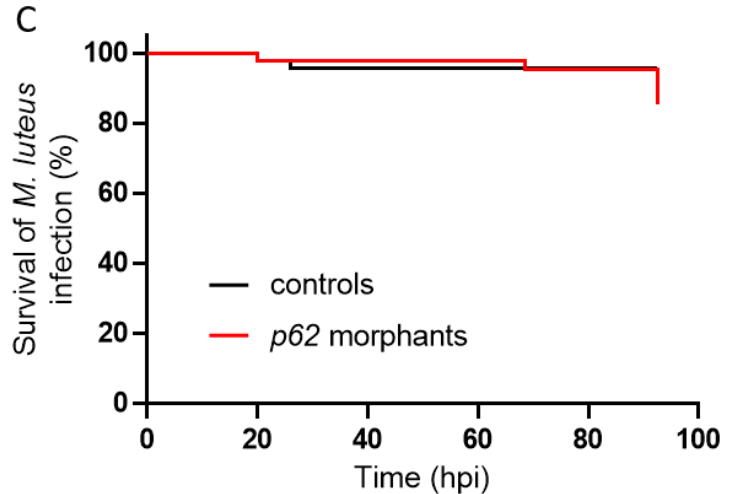

